\title{
The effect of surface tension on the shape of fingers in a Hele Shaw cell
}

\author{
By J. W. MCLEAN AND P. G. SAFFMAN \\ Applied Mathematics, California Institute of Technology, \\ Pasadena, California 91125
}

(Received 24 June 1980)

The experimental results of Saffman \& Taylor (1958) and Pitts (1980) on fingering in a Hele Shaw cell are modelled by two-dimensional potential flow with surface-tension effects included at the interface. Using free streamline techniques, the shape of the free surface is expressed as the solution of a nonlinear integro-differential equation. The equation is solved numerically and the solutions are compared with experimental results. The shapes of the profiles are very well predicted, but the dependence of finger width on surface tension is not quantitatively accurate, although the qualitative behaviour is correct. A conflict between the numerics and a formal singular perturbation analysis is noted but not resolved. The stability of the steady finger to small disturbances is also examined. Linearized stability analysis indicates that the twodimensional fingers are not stabilized by the surface-tension effect, which disagrees with the experimental observations. A possible reason for the discrepancy between theory and experiment is suggested.

\section{Introduction}

It is well known that when two superposed fluids of different densities are accelerated in a direction perpendicular to the surface, the interface will be unstable if the acceleration is from the less dense to the more dense fluid. A similar instability may occur in a porous medium when a less viscous fluid drives a more viscous fluid (Saffman \& Taylor 1958). This instability is responsible for water flooding of oil wells, and is of considerable importance to oil reservoir engineering (Wooding \& Morel-Seytoux 1976).

In order to study this instability, experiments have been performed in a Hele Shaw cell: a channel formed by two closely spaced parallel glass plates which provides a model of two-dimensional flow through a porous medium (Saffman \& Taylor 1958, Pitts 1980). It was observed that the unstable interface forms into a number of 'fingers'. As the flow develops, one of the fingers dominates and suppresses the growth of the others, and the flow reaches a steady state with a single finger propagating without change of shape. $\uparrow$ Our interest will be in the steady-state shape of the finger.

We adopt the notation that $U$ is the velocity of the finger, $2 a$ the lateral width of the channel, $b$ the transverse thickness or spacing $(b / a \ll 1), T$ the interfacial tension between the driven (outside finger) and driving fluids, and $\mu$ is the viscosity of the driven fluid. The parameter $\lambda$ will denote the ratio of the width of the finger to the

† Meng \& Thomson (1978) present results of numerical simulations. 
width of the channel. The adjective lateral will apply to variations in the $x, y$ plane of the plates, whereas transverse will apply to changes across the gap in the $z$ direction.

By neglecting surface tension effects at the free surface, Saffman \& Taylor obtained a closed form solution for the shape of the finger; however, $\lambda$ is undetermined by their analysis. Experiments where the surface tension is negligible $(T / \mu U \ll 1)$ yield $\lambda=\frac{1}{2}$. Comparison of the observed shapes with the Saffman-Taylor solution in these experiments shows excellent agreement. As surface tension effects become important, the finger widens to fill the channel, and the agreement becomes poor.

Several attempts have been made to remove the non-uniqueness of the SaffmanTaylor result. For example, Taylor \& Saffman (1958) gave a number of purely analytical features which singled out the $\lambda=\frac{1}{2}$ solution, but were unable to provide any physical basis for the arguments. Jaquard \& Seguier (1962) presented an exact solution describing the growth of a small circular bubble into fingers with $\lambda=\frac{1}{2}$, but this depends on the initial shape being circular; an initial elliptical shape would give $\lambda \neq \frac{1}{2}$. To our knowledge, there is still no adequate mathematical theory for the width of fingers in a Hele Shaw cell.

A valuable contribution was recently made by Pitts (1980). He put forward the hypothesis that independent of $\lambda$ the finger shapes satisfy $R \sin \hat{\theta}=$ constant, where $R$ is the lateral radius of curvature and $\hat{\theta}$ is the angle between the tangent and the direction of advance of the finger. (It is easily verified that the Saffman-Taylor solution for $\lambda=\frac{1}{2}$ satisfies this relation.) The Saffman-Taylor experiment was repeated and the observed shapes were found to satisfy this relation closely for values of $\lambda$ up to 0.77 . Deviations for $\lambda=0.88$ were attributed to drainage effects and inadequate validity of the Hele Shaw approximations. Pitts proposed further that equating the change in pressure across the meniscus at the nose and sides due to variations of the transverse curvature with the change in pressure due to the flow leads to a unique relation between $\lambda$ and $\mu U a / T b$.

In the experiments carried out by Pitts and most of those by Saffman \& Taylor, the viscosity of the driving fluid was negligible and Pitts' relation is consistent with the observed dependence of $\lambda$ on $\mu U / T$. In particular, $\lambda \rightarrow \frac{1}{2}$ as $\mu U / T$ increases and $\lambda \rightarrow 1$ as $\mu U / T$ decreases. Further experiments are, however, needed to check the $a / b$ dependence. It should also be noted that Pitts' relation involves an unknown constant $m$ describing the difference in transverse curvature at the nose and sides.

The object of this paper is to describe the results of an extension of the SaffmanTaylor analysis to include surface tension effects due to lateral curvature of the interface. Following the assumptions of Saffman \& Taylor, variations in transverse curvature are neglected. The problem is formulated in a potential plane, and the equations for the interface are reduced to a pair of nonlinear integro-differential equations. For finite surface tension, these equations are replaced by a finite difference form and solved numerically using Newton's method. The numerical scheme converged quadratically in all cases to a unique solution for a given value of $\mu U / T$. It appears therefore that the degeneracy of the Saffman-Taylor solutions is removed and the equations have a unique solution for non-zero surface tension. The numerical profiles are compared with experimental photographs, and in all cases show excellent agreement. The qualitative dependence of $\lambda$ on $\mu U / T$ is in agreement with observation; complete quantitative comparison cannot be made because the dependence of the pressure drop across the interface due to lateral curvature depends on the detailed 
structure of the transverse flow and is not known, but the indications are that the agreement is less than satisfactory which suggests that neglect of variations of transverse curvature is questionable. We shall return to this point in $\$ 6$.

The case of small surface tension has also been examined by conventional singular perturbation methods. The strange result is found that it is apparently possible to construct a formally consistent perturbation expansion for arbitrary finger widths, suggesting that the equations for the interface do not have a unique solution. Stringent checks of the numerical procedure were carried out and the internal consistency leads us to believe that the numerical results are correct and the perturbation expansion contains an undetected inconsistency. This conflict with the numerics has not been resolved and raises a problem of considerable mathematical interest.

The effect of the lateral curvature and surface tension on the stability of the steady motion to infinitesimal disturbances has also been examined. The analysis based on the Hele Shaw equations with surface tension neglected predicts that the fingers are unstable, a result which disagrees completely with observation (Taylor \& Saffman 1958). The inclusion of surface tension does not resolve this discrepancy, which suggests that the Hele Shaw equations with the present surface-tension modification are not appropriate for unsteady motion.

\section{Derivation of the equations}

The slow flow of an incompressible fluid in a homogeneous porous medium is governed by Darcy's law and the equation of continuity:

$$
\left.\begin{array}{l}
\mathbf{u}=-\frac{k}{\mu} \nabla p=\nabla \phi, \\
\nabla . \mathbf{u}=\nabla^{2} \phi=0,
\end{array}\right\}
$$

where $u$ is the average velocity vector, $p$ the pressure, $k$ is the permeability of the medium, and $\phi$ is the velocity potential.

Two-dimensional flow in a porous medium may be studied experimentally by means of an analogue developed by Hele Shaw (1898). The motion of an incompressible viscous fluid between two closely spaced parallel plates is such that the components of the mean velocity (averaged across the gap) satisfy:

$$
u=-\frac{b^{2}}{12 \mu} \nabla p=\nabla \phi
$$

(see Lamb 1932, §330). Comparison with (1) shows that the motion in a Hele-Shaw cell is equivalent to two-dimensional flow in a porous medium of permeability $b^{2} / 12$.

We now derive the equations for steady two-dimensional flow produced by a finger along a channel with surface tension effects due to lateral curvature included. It is assumed that the viscosity of the driving fluid is negligible, buoyancy forces are unimportant, and that the driven fluid is completely expelled from the gap by the advancing finger. 'Then the boundary conditions on the (harmonic) velocity potential $\phi$ and pressure $p$ in the driven fluid are

$$
\frac{\partial \phi}{\partial y}=0 \text { on } y= \pm a
$$




$$
\begin{gathered}
\frac{\partial \phi}{\partial n}=U \sin \hat{\theta}, \quad p_{0}-p=\frac{T}{R}+\frac{2 T}{b} \cos \chi \quad \text { on the finger, } \\
\phi \sim 0 \text { as } x \rightarrow-\infty, \quad \lambda a<|y|<a, \\
\phi \sim U \lambda x \text { as } x \rightarrow \infty, \quad-a<y<a
\end{gathered}
$$

where $\chi$ is the contact angle of the meniscus, $p_{0}$ the constant pressure inside the finger, and $p=-12 \mu \phi / b^{2}$. The harmonic conjugate of $\phi$ defines a stream function $\psi$. Following Saffman \& Taylor, we assume further that $\chi=0$, or at least is constant so that the variations of $\phi$ or $p$ along the free boundary are proportional to the lateral curvature. Some of these assumptions can be questioned and some are in fact not valid for the actual experiments. These matters will be discussed later and we shall point out $(\$ 6)$ that the problem with more general assumptions about the flow in the gap can be formulated in this way, with appropriate changes in the values of $\mu$ and $T$, provided the finger occupies a constant fraction $t$ of the stratum between the plates.

It is now convenient to work in a frame of reference moving with the finger with origin at the nose and introduce dimensionless variables (with $a$ and $(1-\lambda) U$ as the units of length and velocity)

$$
\left.\begin{array}{ll}
\hat{x}=(x-U t) / a, & \hat{y}=y / a, \quad \hat{R}=R / a, \\
\hat{\phi}=\frac{\phi-U x}{(1-\lambda) U a}, & \psi=\frac{\psi-U y}{(1-\lambda) U a} .
\end{array}\right\}
$$

The free-boundary potential problem is illustrated in figure 1. Symmetry about the centre-line of the channel has been assumed. The centre-line and the interface is a streamline $(\hat{\psi}=0)$, as is also the wall $\hat{y}=1(\hat{\psi}=-1)$.

The flow region of figure 1 maps into the infinite strip in the potential plane $-\infty<\hat{\phi}<\infty,-1<\hat{\psi}<0$, and a natural formulation is to use Kirchhoff-Helmholtz free streamline theory to study the analytic function $-\log \hat{q}+i \hat{\theta}$, where $\hat{u}-i \hat{v}=\hat{q} e^{-i \hat{\theta}}$ is the (dimensionless) complex velocity relative to the finger, in terms of the complex potential $\hat{w}=\hat{\phi}+i \hat{\psi}$. Note that if $\hat{S}$ measures (dimensionless) arclength along the interface from the nose,

$$
\frac{1}{\hat{R}}=\frac{d \hat{\theta}}{d \hat{S}}=\frac{\partial \hat{\theta}}{\partial \hat{\phi}} \frac{d \hat{\phi}}{d \hat{S}}=\hat{q} \frac{\partial \hat{\theta}}{\partial \hat{\phi}}
$$

so the radius of curvature takes a simple form.

The conformal map

$$
\sigma=s+i t=e^{-\left(\hat{w}-\hat{\phi}_{0}\right) \pi}
$$

maps the potential plane into the upper half $\sigma$-plane, with the upper wall $D E$ going into $-\infty<s<0$, the interface $A B$ into $0<s<1$, and the centreline $B C$ into $1<s<\infty$. On $B C$ and $D E, \hat{\theta}=\pi$. From a standard application of Cauchy's integral theorem, the speed $\hat{q}$ on the interface $A B$ is related to the direction $\hat{\theta}$ by the Cauchy principal-value integral

$$
\log (\hat{q}(s))=-\frac{1}{\pi} P \int_{0}^{1} \frac{\hat{\theta}\left(s^{\prime}\right)-\pi}{s^{\prime}-s} d s^{\prime} .
$$

The constants are fixed by the requirement that $\hat{q} \sim 1$ as $\phi \rightarrow-\infty$. 


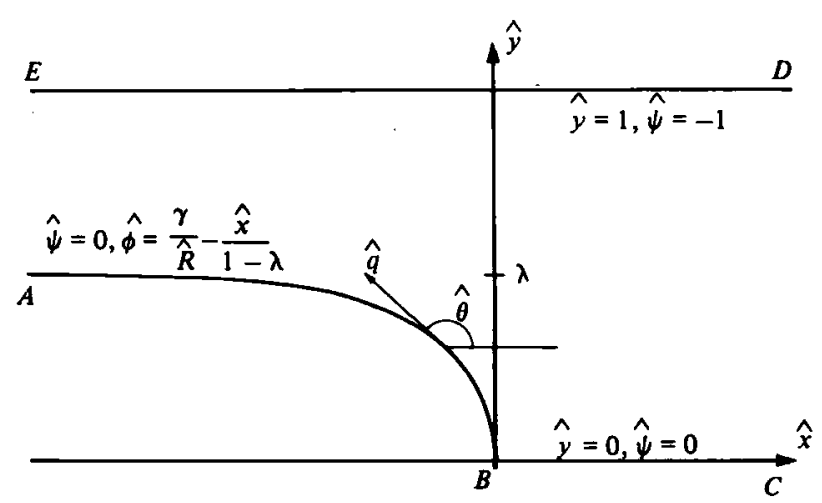

FIGURE 1. The free boundary potential problem in dimensionless variables. $\gamma=T b^{2} / 12 \mu U a^{2}(1-\lambda)$.

This relation for the unknowns $\hat{q}(s)$ and $\hat{\theta}(s)$ comes solely from kinematic considerations. The dynamics are introduced through (4) and (8), which gives

$$
\frac{T b^{2}}{12 \mu U a^{2}} \frac{\hat{q}}{1-\lambda} \frac{\partial \hat{\theta}}{\partial \hat{\phi}}-\frac{\hat{x}}{1-\lambda}=\hat{\phi}, \quad \hat{\phi}_{0}<\hat{\phi}<\infty
$$

Using $s$ as the independent variable and the relations

$$
\cos \hat{\theta}=\frac{d \hat{x}}{d \hat{\hat{S}}}, \frac{d \hat{\phi}}{d \hat{S}}=\hat{q}, \quad \frac{d \hat{\phi}}{d s}=-\frac{1}{\pi s},
$$

we differentiate (11) along the interface to obtain the second relation between $\hat{q}$ and $\hat{\theta}$,

$$
\frac{T b^{2} \pi^{2}}{12 \mu U a^{2}} \hat{q} s \frac{d}{d s}\left(\hat{q} s \frac{d \hat{\theta}}{d s}\right)-(1-\lambda) \hat{q}=\cos \hat{\theta}, \quad 0<s<1 .
$$

The appropriate boundary conditions for (10) and (13) are

$$
\left.\begin{array}{ll}
\hat{\theta}(0)=\pi, & \hat{q}(0)=1 /(1-\lambda), \\
\hat{\theta}(1)=\pi / 2, & \hat{q}(1)=0
\end{array}\right\}
$$

corresponding to uniform flow at the tail $A(s=0)$ and a stagnation point at the nose $B(s=1)$.

Our task is to solve equations (10) and (13) subject to the boundary conditions (14) for given values of $U, \mu, T, a, b$, and determine if $\lambda$ is then uniquely determined. Since from (10) and (14)

$$
\log (1-\lambda)=\frac{1}{\pi} \int_{0}^{1} \frac{\hat{\theta}-\pi}{s^{\prime}} d s^{\prime}
$$

we can rewrite the equations in a form which does not explicitly involve $\lambda$. Introducing

we have

$$
\begin{gathered}
\theta=\hat{\theta}-\pi, \quad q=(1-\lambda) \hat{q} \\
\kappa=\frac{T b^{2} \pi^{2}}{12 \mu U a^{2}(1-\lambda)^{2}}
\end{gathered}
$$

$$
\log q(s)=-\frac{s}{\pi} P \int_{0}^{1} \frac{O\left(s^{\prime}\right)}{s^{\prime}\left(s^{\prime}-s\right)} d s^{\prime}
$$


with

$$
\kappa q s \frac{d}{d s}\left(q s \frac{d \theta}{d s}\right)-q=-\cos \theta
$$

$$
\theta(0)=0, \quad q(0)=1, \quad \theta(1)=-\pi / 2, \quad q(1)=0 .
$$

If these equations have a unique solution for given $\kappa$, then the width of the finger is uniquely determined by (15) to give $\lambda$ as a function of $T b^{2} / \mu U a^{2}$. Note that this result is qualitatively different from that of Pitts, whose argument gives $\lambda$ as a function of $T b / \mu U a$. Further experiments with channels of varying gap should distinguish between the theories.

The shape of the interface follows from

and is given by

$$
\frac{d \hat{z}}{d \hat{w}}=\frac{e^{i \hat{\theta}}}{\hat{q}}
$$

$$
\hat{x}(s)+i \hat{y}(s)=-\frac{(1-\lambda)}{\pi} \int_{s}^{1} \frac{e^{i \theta}}{s q} d s .
$$

The value of $\lambda$ can also be obtained by evaluating $\hat{y}(0)$, and provides a check on the accuracy of the computations.

In these variables, the Saffman-Taylor solution is

$$
q_{0}=\left(\frac{1-s}{1+s \alpha}\right)^{\frac{1}{2}}, \quad \theta_{0}=\cos ^{-1} q_{0},
$$

where $\alpha=(2 \lambda-1) /(1-\lambda)^{2}$ is arbitrary. This solution can be shown to satisfy (18) for $\kappa=0$ (McLean 1980).

\section{Endpoint singularity}

Before proceeding with the numerical computations, it is necessary to analyse the possible singularities of the solution. For $\kappa=0$, the solution has square-root singularities at both endpoints. For non-zero surface tension we seek a solution about $s=0$ of the form

$$
\left.\begin{array}{l}
\theta(s)=a_{1} s^{\tau}+a_{2} s^{2 \tau}+\ldots . \\
q(s)=1+b_{1} s^{\tau}+b_{2} s^{2 \tau}+\ldots .
\end{array}\right\}
$$

This expansion is substituted into (18). Using the result

$$
P \int_{0}^{1} \frac{x^{\tau}}{x-y} d x=\frac{1}{\tau}+\frac{y}{\tau-1}-\pi y^{\tau} \cot \pi \tau+O\left(y^{2}\right)
$$

for $0<\tau<1$, we equate the coefficients of the $s^{\tau}$ term, giving

From (19) we obtain

$$
b_{1}=a_{1} \cot \pi \tau \text {. }
$$

$$
b_{1}=\kappa \tau^{2} a_{1} \text {. }
$$

Eliminating $b_{1}$ and requiring that $a_{1} \neq 0$, we find that the exponent $\tau$ is given by

$$
\frac{1}{\tau^{2}} \cot \pi \tau=\kappa
$$


which has a root with $0<\tau<1$. The coefficients $b_{1}, b_{2}$, and $a_{2}$ can be found recursively as functions of $a_{1}$. As $\kappa \rightarrow 0, \tau \rightarrow \frac{1}{2}$ which agrees with the Saffman-Taylor solution (23).

It can be shown (McLean 1980) that terms linear in $s$ in the expansion generate singularities from (18) of the form $s \log ^{n} s$ which cannot satisfy the equation and so are not present. Since the cotangent is periodic, (25) has multiple solutions $\left\{\tau_{n}\right\}$. A proper expansion must account for these extra terms, and the full local expansion is of the form

$$
\theta(s)={ }_{1} a_{1} s^{\tau_{1}}+{ }_{1} a_{2} s^{2 \tau_{1}}+\ldots+{ }_{2} a_{1} s^{\tau_{2}}+{ }_{2} a_{2} s^{2 \tau_{2}}+\ldots
$$

At the nose $(s=1)$ an expansion in powers of $(1-s)^{\frac{1}{2}}$ can be shown to be consistent with the equations as required by symmetry. But in this case, the coefficients cannot be found recursively, but are given globally as integrals of $\theta$ over the entire interval. For details, see McLean (1980).

\section{Numerical treatment}

We change the independent variable so that $q$ and $\theta$ will be differentiable at the endpoints. If

$$
\zeta=\left(1-s^{\tau}\right)^{\frac{1}{2}}, \quad s^{\tau}=1-\zeta^{2}
$$

the variables have at least two derivatives with respect to $\zeta$ at the end points. To evaluate the principal value integral, the singular part of the integrand is subtracted off and (18) is written

$$
\log q=\frac{1}{\pi}\left\{\int_{0}^{1} \frac{\theta\left(s^{\prime}\right)}{s^{\prime}} d s^{\prime}-\int_{0}^{1} \frac{\theta\left(s^{\prime}\right)-\theta(s)}{s^{\prime}-s} d s^{\prime}-\theta(s) \log \left(\frac{1-s}{s}\right)\right\} .
$$

The system is written in terms of $\zeta$ with derivatives replaced by a three point centred difference quotient and the integral evaluated using the trapezoidal rule. The interval $[0,1]$ is broken into $N$ equal segments of length $h=1 / N$. Newton's method is used to solve the finite difference equations for $\theta$ at the $N-1$ interior mesh points. The numerical scheme was coded in vectorized FORTRAN and computed on the CDC CYBER 203 in Minneapolis. The Newton method is said to have converged when the maximum residual is less than $10^{-10}$ (generally 4-5 iterations, taking about $0 \cdot 2$ seconds CPU time for $N=50$ ). After a converged solution is obtained, the Cartesian coordinates are given by evaluation of equation (22).

Newton's method is particularly valuable for this problem because of the singularity of the system when $\kappa=0$. If the solutions are not unique for $\kappa>0$, then the Jacobian of the Newton iteration should show singular behaviour. For the first attempt to find a solution for $\kappa \neq 0$, we used the Saffman-Taylor solution (23) for $\lambda=\frac{1}{2}$ as the first guess and took $\kappa=0.237(\tau=0.48)$. The Newton iteration converged quadratically with no sign of singular behaviour. Continuation was employed to construct a unique family of solutions for values of $\kappa$ from 0.064 to $4 \times 10^{7}$ and the shapes were computed.

The numerical profiles are compared with the experimental results of Pitts (1980) in figures $2(a), 2(b)$, and $2(c)$, for values of $\kappa$ chosen so that the calculated $\lambda$ is equal to the observed $\lambda$. The agreement in profile shape is remarkably good.

Numerous tests were performed to check the numerical procedure. The number of mesh points was varied between 25 and 200. Examination of the results indicated that the error was $O\left(h^{2}\right)$ as expected from the trapezoidal rule. The results were independent 

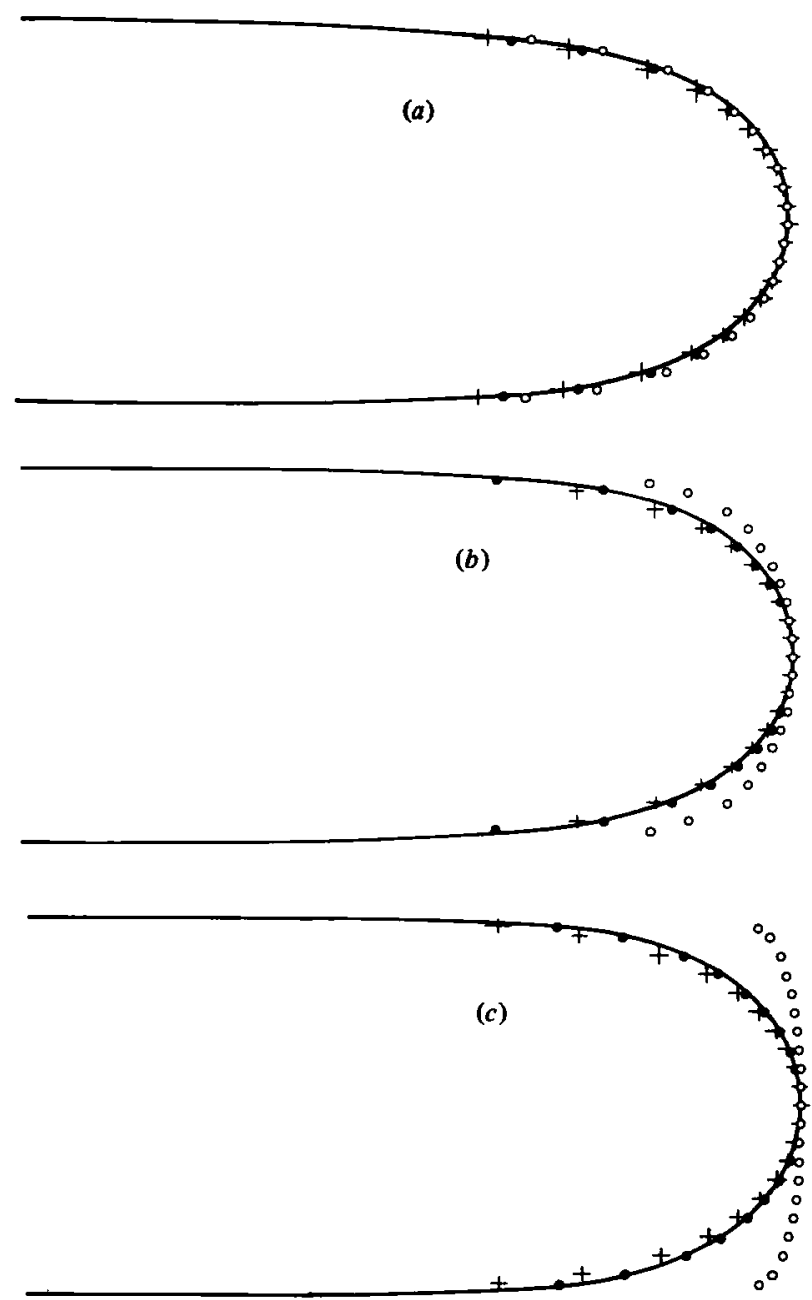

FigURE 2. Comparison of theory and experiment. (a) $\lambda=0.54,(b) \lambda=0.68,(c) \lambda=0.88$. Solid line, experiment (Pitts 1980); $O$, Saffman \& Taylor solution; + , Pitts theory; $O$, numerical results.

of the initial guess, provided this was not so bad that the iteration diverged. The convergence was always quadratic, provided $\kappa$ was neither too small nor too large. The Jacobian determinant is shown in figure 3 for $N=50$. The behaviour is consistent with isolated solutions for $\kappa>0$ and singular behaviour when $\kappa=0$.

Doubts raised by a conflict of these results with a perturbation expansion $(\$ 5)$ suggested some additional tests; e.g. fixing the slope at $s=0$ (i.e. giving $a_{1}$ in (24)) and solving (18) and (19) on a reduced interval, or fixing $\lambda$ and solving an inflated system based on (15), (18), and (19). Both these tests confirm the previous calculations.

In short, all indications of the numerical investigation are that the system defined by (18), (19), and (20) has a unique solution for $\kappa>0$. Values of $\lambda$ for various $\kappa$ are listed in table 1 and plotted in figure 4. Also in table 1 are the corresponding values of $\mu U / T$ for the Saffman-Taylor and Pitts value of $a / b=15 \cdot 875$. The comparison of calculated with observed values will be discussed later. 


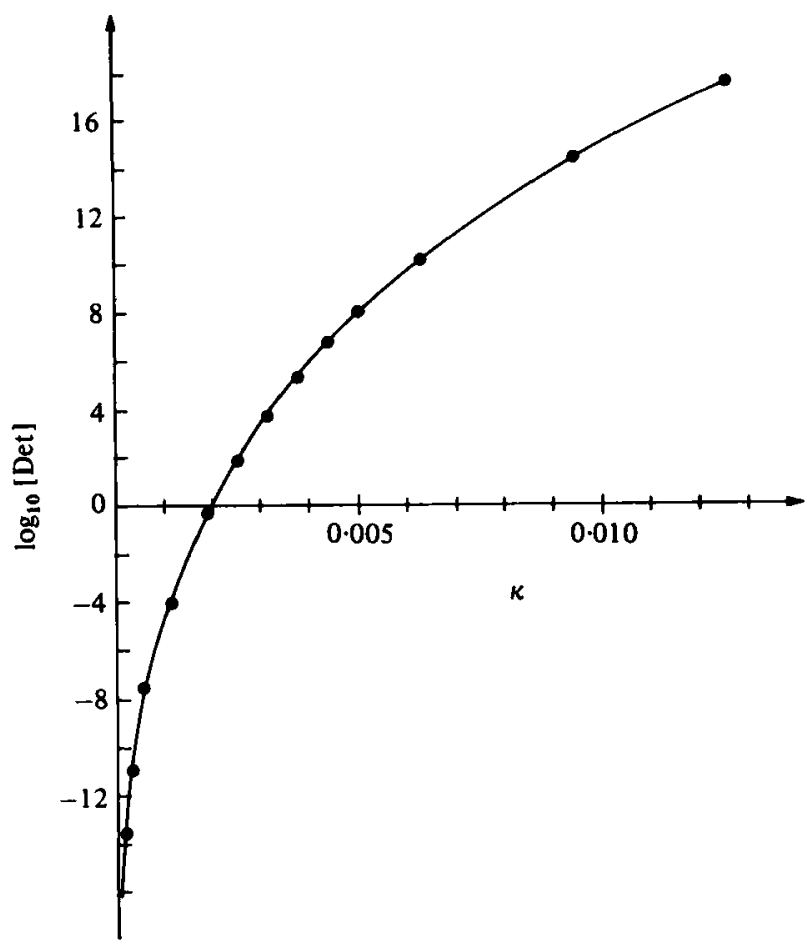

Figure 3. Determinant of the Jacobian versus the surface tension parameter, indicating singular behsviour as $\kappa \rightarrow 0$.

\section{Perturbation expansions}

For $\kappa \ll 1$, the system would appear suitable for investigation by standard singular perturbation methods. A formal perturbation expansion was constructed by the method outlined here. Since the behaviour of $\theta$ near $s=0$ is non-uniform in $\kappa$, we construct an 'inner-outer' matched asymptotic expansion. The outer expansion is taken to be

$$
\left.\begin{array}{l}
\theta(s ; \kappa)=\theta_{0}(s)+\kappa \theta_{1}(s)+\kappa^{2} \theta_{2}(s)+\ldots, \\
q(s ; \kappa)=q_{0}(s)+\kappa q_{1}(s)+\kappa^{2} q_{2}(s)+\ldots,
\end{array}\right\}
$$

where the leading term $\left(q_{0}, \theta_{0}\right)$ is the Saffman-Taylor solution (23). Substituting the expansion (28) in the equations (18) and (19) and matching terms linear in $\kappa, \theta_{1}(s)$ is found to satisfy a singular linear integral equation

$$
\frac{s}{\pi} P \int_{0}^{1} \frac{\theta_{1}\left(s^{\prime}\right)}{s^{\prime}\left(s^{\prime}-s\right)} d s^{\prime}=\theta_{1} \tan \theta_{0}-s \frac{d}{d s}\left(q_{0} s \frac{d \theta_{0}}{d s}\right) .
$$

The solution of (29) can be calculated explicitly (Carrier, Krook \& Pearson 1966) and is found to be

$$
\begin{aligned}
& \theta_{1}(s)=\frac{(1+\alpha)^{\frac{1}{2}}}{\pi} \frac{(s(1-s))^{\frac{1}{2}}}{1+s \alpha}\left\{A+\frac{\pi(1+\alpha)^{\frac{1}{2}}}{4} \frac{s^{\frac{1}{2}}(1-2 s \alpha)}{(1+s \alpha)^{\frac{s^{2}}{2}}}+\frac{1}{2(1+s \alpha)^{2}}\right. \\
& \left.\left[\frac{1+2 \alpha-s \alpha(\alpha+2)}{\alpha+1}-\frac{(1-s)^{\frac{1}{2}}(2 \alpha s-1)}{2(1+\alpha s)^{\frac{1}{2}}} \log \frac{s(1+\alpha)}{2\{(1-s)(1+\alpha s)\}^{\frac{1}{2}}+(\alpha-1) s+2}\right]\right\},
\end{aligned}
$$




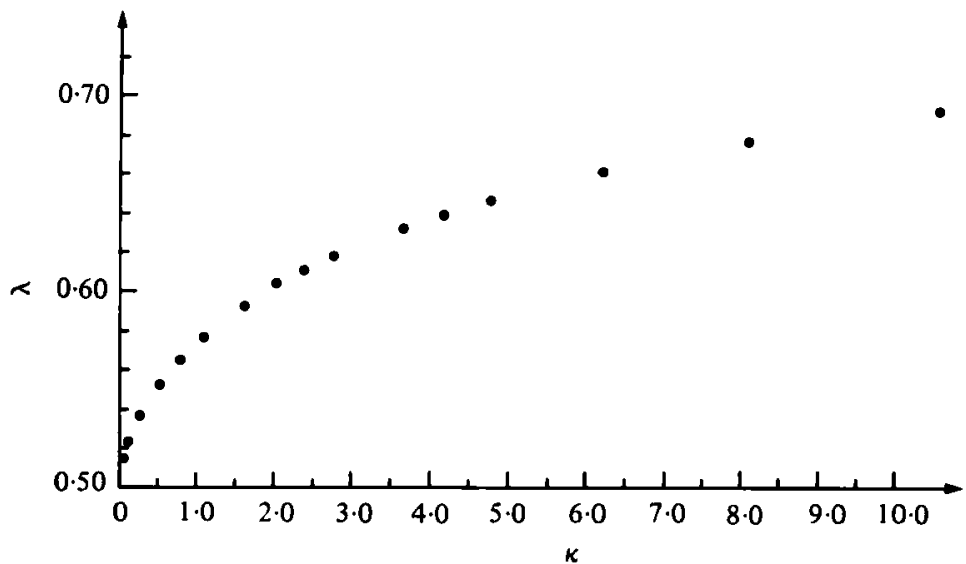

Figure 4. The finger width $\lambda$ as a function of the surface-tension parameter $\kappa$.

\begin{tabular}{cccc}
\hline$\tau$ & $\kappa$ & $\mu U / T$ & $\lambda$ \\
0.495 & 0.069 & 0.2168 & 0.515 \\
0.49 & 0.131 & 0.1098 & 0.524 \\
0.48 & 0.273 & 0.0557 & 0.537 \\
0.46 & 0.597 & 0.0279 & 0.557 \\
0.43 & 1.21 & 0.0154 & 0.582 \\
0.40 & 2.03 & 0.0102 & 0.604 \\
0.35 & 3.09 & 0.0082 & 0.640 \\
0.30 & 8.07 & 0.0039 & 0.678 \\
0.25 & 16.0 & 0.0026 & 0.720 \\
0.20 & $\mathbf{3 4 . 4}$ & 0.0017 & 0.767 \\
0.16 & $\mathbf{7 1 . 1}$ & 0.0013 & 0.809 \\
0.10 & $\mathbf{3 0 8}$ & 0.0008 & 0.881 \\
0.05 & $\mathbf{2 5 2 6}$ & 0.0005 & 0.947 \\
0.02 & $\mathbf{3 9 7 4 0}$ & 0.0003 & 0.984
\end{tabular}

TABLe 1

where the constant $A$ is an arbitrary parameter. Each succeeding term in the expansion (28) satisfies on equation of the form (29) with a different inhomogeneous term. This equation can be solved provided the inhomogeneous term vanishes at $s=0$, which was always found to be the case.

The outer expansion (28) is expanded about $s=0$, and the inner expansion (24) is expanded in powers of $\kappa$. The coefficient $a_{1}$ is chosen to match the $s^{\frac{1}{2}}$ terms. The remaining terms are then compared to check consistency. We have verified that the $s^{\frac{1}{2}} \log s$ terms match at $O(\kappa)$, the $s^{\frac{1}{2}} \log ^{2} s$ terms match at $O\left(\kappa^{2}\right)$, the $s$ terms match at $O(\kappa)$, and the $s^{n+\frac{1}{2}}$ terms match to all orders. To the order considered, $\alpha$ and $A$, and hence $\lambda$ remain undetermined. For full details, see McLean (1980).

The perturbation analysis suggests that the system defined by equations (18), (19), and (20) does not have a unique solution, a conclusion in conflict with the numerical results. It is possible that the perturbation expansion is inconsistent at higher order or that transcendentally small terms are present, but we detected no reason why this should happen. The fact that the difficulty is subtle is suggested by the finding that 
the choice of $A$ and $\alpha$, to give a width in agreement with the numerics, leads to values of $\theta_{0}+\kappa \theta_{1}$ which agree with the computed values of $\theta$ for small $\kappa$ to within the accuracy of the computations. Despite considerable effort, the apparent failure of the perturbation expansion to fix a unique $\lambda$ remains unexplained.

An attempt was also made to construct a perturbation expansion for $\kappa \gg 1$, in order to find the limiting solution as $\kappa \rightarrow \infty$ or determine if there is an upper bound on $\kappa$ for solutions of the assumed type to exist. We were unable to construct a consistent expansion or answer this question. Numerical solutions were found for $\kappa=O\left(10^{7}\right)$; the values of $\lambda$ were about 0.99 and the profiles were smooth to the eye. For larger values the numerics became unreliable due to the appearance of large gradients of $q$ and $\theta$ at the endpoints.

\section{The dependence of finger widths on surface tension}

The equations that we have studied are based on the boundary conditions (4), which in turn are based on the hypothesis that the driving fluid completely expels the driven fluid. In reality, it is expected that a thin film of the driven fluid will be left adhering to the plates and that the driving fluid penetrates the gap in a tongue of thickness $b t$, where $t$ can be expected to be a function of $\mu U / T$. For the case of a viscous fluid expelled from a tube by a finger of less viscous fluid advancing with speed $U$, it was found experimentally that (Fairbrother \& Stubbs 1935; Taylor 1961)

$$
t=1-(\mu U / T)^{\frac{1}{2}}, \quad 0<\mu U / T<0.09 .
$$

A similar dependence can be expected in a Hele-Shaw experiment. Now it was shown by Saffman \& Taylor that the motion when the advancing finger fills a constant fraction $t$ of the stratum is similar to that when the driven fluid is assumed completely expelled and has viscosity $A \mu$, where

$$
A=t+\frac{\mu_{2}(1-t)^{2}\left(1+\frac{1}{2} t\right)}{\mu t+\frac{3}{2} \mu_{2}\left(1-t^{2}\right)}
$$

and $\mu_{2}$ is the viscosity of the driving fluid.

The pressure drop across the interface depends on the details of the flow near the nose of the tongue, and cannot apparently be found without-a full analysis of the three-dimensional flow about the advancing tongue. All we can safely conclude, essentially from dimensional analysis, is that there should be a relation of the form

$$
\Delta p=\frac{T}{b} f(t)+\frac{T}{R} g(t),
$$

where $f$ and $g$ are unknown functions of the tongue width $t$ which in turn is some function of $\mu U / T$. However, if we assume that $t$ is constant, then the problem that we have solved is equivalent to the real one with

$$
\kappa=\frac{g}{A} \frac{T b^{2} \pi^{2}}{12 \mu U a^{2}(1-\lambda)^{2}} .
$$

In other words, finite tongue thickness is just equivalent to increasing the surface tension by a factor $g / A$, provided it is constant. 


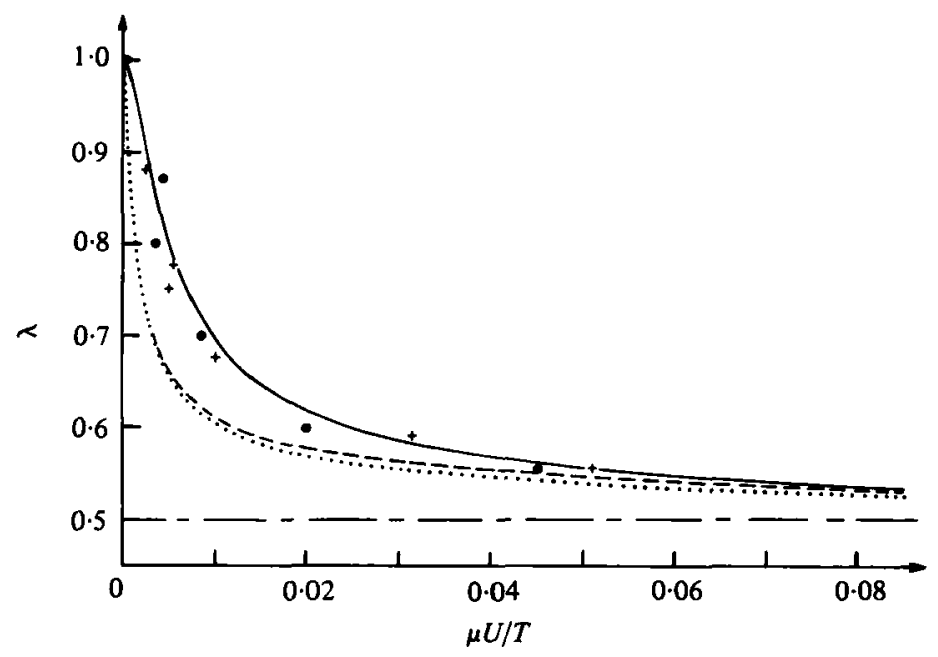

Fraure 5. Finger width $\lambda$ versus $\mu U / T$. + , Pitts' experimental results; 9 , Saffman \& Taylor experimental results; —_, Pitts' relation; $\cdots$, numerical results; ---, numerics adjusted for finite tongue thickness.

In figure 5, we plot the relation between $\lambda$ and $\mu U / T$. The upper solid line is the empirical relation of Pitts (1980), which agrees well with both Pitts' and Saffman \& Taylor's experimental observations. T The current results are indicated by the lower dotted line. The uncorrected theoretical values $(g / A=1)$ differ significantly from the experimental values. Corrected values, shown by the dashed line, were found as follows. From figure 4 or table 1, we have $\lambda v s$. $\kappa$. These were substituted into (34) with $g=1$ and $A=t$ (the appropriate value for $\mu_{2} / \mu \ll 1$ ) and combined with (31) to give $\mu U / T$ as a function of $\lambda$. The improvement is slight. Thus, the quantitative dependence of $\lambda$ on $\mu U / T$ is unsatisfactory, unless $g$ is greater than 1 , or $t$ is less than the value suggested by (31) which is for experiments in tubes.

An alternative possibility is that $t \neq$ constant. Indeed, the $U$ in (31) should strictly have been the normal velocity of the interface, which is not constant. Then the variations in $f(t)$ would control the shape. This, of course, is essentially Pitt's suggestion. The theoretical problem now becomes far more difficult, but it is hoped to study this possibility in further work. As mentioned earlier, experiments on the dependence of $\lambda$ on $\mu U / T$ for various $a / b$ would help establish the relative importance of the two terms in (33).

\section{Stability analysis}

Taylor \& Saffman (1958) examined the stability of the fingers neglecting surface tension. Here, the lateral surface tension effects are included. Also, we suppose that the tongue completely fills the gap. In this section, $t$ denotes time, and we use dimensional variables with $\phi$ and $\psi$ denoting the velocity potential and streamfunction in coordinates moving with the finger.

$\dagger$ Weare grateful to Dr Pitts for pointing out that the data of Saffman \& Taylor were incorrectly plotted in figure 14 of the original paper and that the correct figure is to be found in vol. IV of Taylor's collected papers, p. 328. 
In the physical plane, the steady finger is bounded by the streamline $\psi=0$. The motion in oscillations about the steady shape is described in the potential plane by

$$
\begin{aligned}
& x=x_{0}(\phi, \psi)+\epsilon \xi(\phi, \psi, t), \\
& y=y_{0}(\phi, \psi)+\epsilon \eta(\phi, \psi, t) .
\end{aligned}
$$

The interface is given by an equation

$$
G(\phi, \psi, t) \equiv \psi-\epsilon F(\phi, t)=0,
$$

where $\xi, \eta$, and $F$ are the perturbations about the steady motion. The free surface $G=0$ is a material surface moving with the fluid, so the kinematic condition is:

$$
\frac{D}{D t}(\psi-\epsilon F)=0 \text { on } G=0,
$$

where $D / D t$ is the convective derivative. The stability analysis will be carried out in the potential plane, so the kinematic condition must be written with $(\phi, \psi)$ as independent variables (see Longuet-Higgins 1978). Retaining terms linear in the perturbations, the boundary condition becomes

$$
\boldsymbol{F}_{t}+q^{2} \boldsymbol{F}_{\phi}+q \cos \hat{\theta} \eta_{t}-q \sin \hat{\theta} \xi_{t}=0
$$

on the undisturbed finger $\psi=0, \phi_{0}<\phi<\infty$.

The second condition at the interface is the pressure boundary condition. The lateral curvature has the parametric representation

$$
\frac{1}{R}=\left|\frac{G_{x x} G_{y}^{2}-2 G_{x} G_{y} G_{x y}+G_{y y} G_{x}^{2}}{\left(G_{x}^{2}+G_{y}^{2}\right)^{\frac{8}{2}}}\right| .
$$

In the potential plane, this becomes, to $O(\epsilon)$,

$$
\frac{1}{R}=q \hat{\theta}_{\phi}+\epsilon\left(q F_{\phi \phi}-q_{\phi} F_{\phi}\right)
$$

Retaining terms linear in the perturbation, the dynamic boundary condition to be satisfied on $\psi=0$ becomes

$$
\frac{T b^{2}}{12 \mu U}\left\{F\left(q \hat{\theta}_{\phi}^{2}-q_{\phi \phi}+q_{\phi}^{2} / q\right)+q F_{\phi \phi}-q_{\phi} F_{\phi}\right\}-\xi+F \frac{\sin \hat{\theta}}{q}+g(t)=0
$$

where $g(t)$ is an arbitrary function of time.

The entire potential plane $-U a(1-\lambda)<\psi<U a(1-\lambda)$ is mapped to a semiinfinite strip $-\pi / 2<u<\pi / 2, v>0$ through the transformations

$$
\begin{aligned}
& w=\phi+i \psi=-\frac{U a(1-\lambda)}{\pi} \log \left(1-Z^{2}\right)+\phi_{0}, \\
& Z=\sin \Omega=\sin (u+i v),
\end{aligned}
$$

where the $u$ axis corresponds to all the interface, both above and below the centreline.

The perturbation $\xi+i \eta$ must be an analytic function of $\Omega$. For a symmetric perturbation, we choose the representation

$$
\xi+i \eta=e^{\sigma t} \sum_{0}^{\infty} a_{n} e^{2 n i \Omega}, \quad W \equiv F / q=e^{\sigma t} \sum_{0}^{\infty} b_{n} \cos (2 n-1) u .
$$


The real coefficients $\left\{a_{n}, b_{n}\right\}$ are chosen to satisfy (35) and (36) on $v=0$, and the condition that this can be done determines the eigenvalue $\sigma$.

For no surface tension $(T=0)$, the equations can be solved in closed form. Using (37) in (35) and (36), and equating Fourier coefficients, one can show that the series is finite if $\sigma$ is one of the values

$$
\sigma_{n}=2 \pi n U / a, \quad n=0,1,2, \ldots .
$$

The corresponding eigenfunction includes the first $n+1$ terms of (37). In particular, $n=0$ corresponds to a translation of the finger, while $n=1$ is the perturbation

$$
\xi+i \eta=e^{2 \pi t U / a}\left(1+\frac{1}{3} e^{2 i \Omega}\right) .
$$

This result was first obtained by Taylor \& Saffman (1958). Since the eigenvalues $\sigma_{n}$ are positive, the analysis suggests that the fingers are unstable, a conclusion in complete disagreement with the experimental observations, which show not the slightest trace of instability.

To follow these eigenvalues to finite surface tension, equations (35) and (36) are written using $\zeta$ as an independent variable. The series (37) is truncated at a finite number of terms $N$. The coefficients $\left\{a_{n}, b_{n}\right\}$ are chosen to satisfy (35) and (36) at $N$ interior mesh points, yielding the linear system

$$
\left(A_{N}-\sigma B_{N}\right)\left(\begin{array}{l}
a_{n} \\
b_{n}
\end{array}\right)=0 .
$$

$A_{N}$ and $B_{N}$ are square matrices of order $2 N \times 2 N$. Newton's method is used to compute the eigenvalues for $T>0$. The truncation $N$ is varied to gain confidence in the solution. Using $N=\{5,6,8,10,12,15\}$, the lowest eigenvalue did not vary more than $1 \%$. The lowest eigenvalue was computed for $0<\kappa<1$. It remains real and positive, decreasing by about $10 \%$ from its value of $2 \pi U / a$ for $\kappa=0$, indicating the finger is unstable and that surface tension associated with lateral curvature does not stabilize the finger. This is a further indication that variations in the gap may be crucial. For full details of the stability analysis, again see McLean (1980).

\section{Other steady solutions}

Although the inclusion of surface tension in the manner considered here has apparently removed the non-uniqueness of the solution (apart from the unresolved conflict between the numerics and the perturbation analysis), it is worth noting that there still exist a continuum of steady solutions for given $U$ and $T$ if the interface stretches entirely across the channel. For suppose in channel fixed co-ordinates the interface has the equation

$$
f(x, y, t) \equiv U t+\eta(y)-x=0, \quad-a \leqslant y \leqslant a,
$$

and let us again suppose the tongue thickness is constant so that we may assume the driving fluid completely expels the driven. Then Darcy's law and the kinematic and dynamic boundary conditions are satisfied by $\phi_{x}=U$ in each fluid if

$$
\left(\mu-\mu_{2}\right) U \eta+\frac{T b^{2}}{12} \frac{\eta_{y v}}{\left(1+\eta_{\nu}^{2}\right)^{\frac{3}{2}}}=f n(t) .
$$


Thus any $\eta(y)$ which satisfies the equation

$$
\eta_{y y}+\frac{12 U}{T b^{2}}\left(\mu-\mu_{2}\right)\left(1+\eta_{y}^{2}\right)^{\frac{3}{2}} \eta=0, \quad-a \leqslant y \leqslant a
$$

is a possible interface, provided only that $|\eta|<\infty$. The curious feature here is that the degeneracy vanishes when $T=0$, as then $\eta=0$ is the only solution.

This work was supported by the Department of Energy (Office of Basic Energy Sciences). We also wish to thank Control Data Corporation for the granting of time on their CYBER 203 computer at the C.D.C. Service Center, Arden Hills, Minnesota, which enabled us to implement Newton's method for large matrices.

\section{REFERENCES}

Carrier, G. F., Krook, M. \& Pearson, C. E. 1966 Functions of a Complex Variable. McGraw: Hill.

Fairbrother, F. \& StubBs, A. E. 1935 The 'bubble tube' method of measurement. J. Chem. Soc. 1, 527-530.

HeLe-SHAw, H. J. S. 1898 On the motion of a viscous fluid between two parallel plates. Nature 58, 34-36.

JACQUARD, P. \& SÉguinR, P. 1962 Mouvement de deux fluides en contact dans un milieu poreux. J. Méc. 1, 367-394.

Lamb, H. 1932 Hydrodynamics, 6th edn. Cambridge University Press.

Longuet-Higarns, M. S. 1978 The instabilities of gravity waves of finite amplitude in deep water. Proc. Roy. Soc. A 360, 471-488.

Mclean, J. W. 1980 The fingering problem in flow through porous media. Ph.D. dissertation. California Institute of Technology.

Meng, J. C. S. \& Thomson, J. A. L. 1978 Numerical studies of some nonlinear hydrodynamic problems by discrete vortex element methods. J. Fluid Mech. 84, 433-453.

Pitrs, E. 1980 Penetration of fluid into a Hele Shaw cell. J. Fluid Mech. 97, 53-64.

SaffMan, P. G. \& TAYLor, G. I. 1958 The penetration of fluid into a porous medium or HeleShaw cell. Proc. Roy. Soc. A 245, 312-329.

TAYlor, G. I. 1961 Deposition of a viscous fluid on the wall of a tube. J. Fluid Mech. 10, 161165.

TAYloR, G. I. \& SAFrman, P. G. 1958 Cavity flows of viscous fluids in narrow spaces. $2 n d$ Symp. on Naval Hydrodynamics.

Wooding, R. A. \& Morel-SeYtoux, H. J. 1976 Multiphase fluid flow through porous media. Ann. Rev. Fluid Mech. 8, 233-274. 